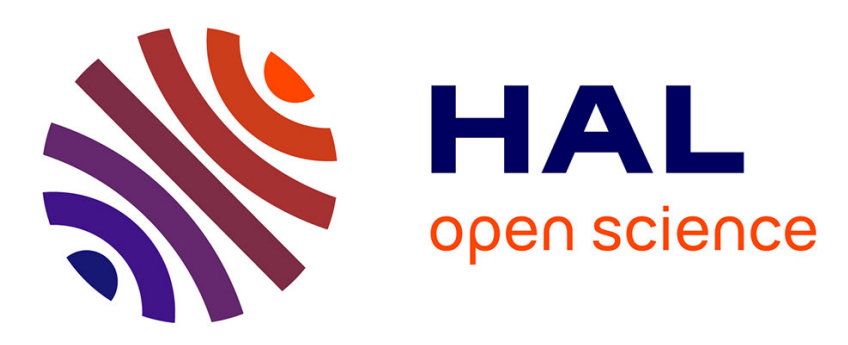

\title{
Texture classification based on the generalized gamma distribution and the dual tree complex wavelet transform
}

\author{
Ahmed Drissi El Maliani, Nour-Eddine Lasmar, Mohammed El Hassouni, \\ Yannick Berthoumieu
}

\section{To cite this version:}

Ahmed Drissi El Maliani, Nour-Eddine Lasmar, Mohammed El Hassouni, Yannick Berthoumieu. Texture classification based on the generalized gamma distribution and the dual tree complex wavelet transform. ISIVC - International Symposium on Image/Video Communications over fixed and mobile networks, 2010, Rabat, Morocco. pp.1-4, 10.1109/ISVC.2010.5656257 . hal-00727115

\section{HAL Id: hal-00727115 \\ https://hal.inria.fr/hal-00727115}

Submitted on 2 Sep 2012

HAL is a multi-disciplinary open access archive for the deposit and dissemination of scientific research documents, whether they are published or not. The documents may come from teaching and research institutions in France or abroad, or from public or private research centers.
L'archive ouverte pluridisciplinaire HAL, est destinée au dépôt et à la diffusion de documents scientifiques de niveau recherche, publiés ou non, émanant des établissements d'enseignement et de recherche français ou étrangers, des laboratoires publics ou privés. 


\title{
Texture Classification Based On The Generalized Gamma Distribution And The Dual Tree Complex Wavelet Transform
}

\author{
Ahmed Drissi El Maliani ${ }^{a}$, Nouredine Lasmar ${ }^{b}$, Mohammed El Hassouni $^{c}$, Yannick Berthoumieu $^{b}$ \\ ${ }^{a}$ LRIT UA to CNRST, Faculty of sciences, BP 1014 Rabat, Morroco \\ ${ }^{b} I M S$ - Groupe Signal, UMR 5218 CNRS, ENSEIRB, Université de Bordeaux, France \\ ${ }^{c}$ DESTEC, FLSHR, Rabat, Morroco \\ Emails: meliani.ahmed@hotmail.fr,nour-eddine.lasmar@ims-bordeaux.fr,mohamed.elhassouni@gmail.com, \\ yannick.berthoumieu@ims-bordeaux.fr
}

\begin{abstract}
This paper deals with stochastic texture modeling for classification issue. A generic stochastic model based on three-parameter Generalized Gamma (GG) distribution function is proposed. The GG modeling offers more flexibility parameterization than other kinds of heavy-tailed density devoted to wavelet empirical histograms characterization. Moreover, Kullback-leibler divergence is chosen as similarity measure between textures. Experiments carried out on Vistex texture database show that the proposed approach achieves good classification rates.
\end{abstract}

\section{INTRODUCTION}

Coming up with accurate texture representation is central in computer vision applications such as covering texture retrieval, texture synthesis and texture classification. Considering the literature, recent works point out that the marginal stochastic modeling approach is well suited for texture retrieval. It exhibits demonstrated effectiveness properties and its formulation simplicity contributes to its popularity for characterizing empirical wavelet histograms. The Generalized Gaussian density (GGD), proposed in [1], has a considerable notoriety. Do et al. in their pioneering work proposed the GGD model for characterizing histograms of subbands issued from the Discrete Wavelet Transform (DWT). Recently, other kinds of decomposition appeared such as Dual Tree Complex Wavelet Transform (DTCWT) [2]. This latter decomposition has many advantages in terms of shift invariance, rotation invariance and directional selectivity offering six orientations in each decomposition level. In the case of complex coefficients characterization, Weibull and Gamma distributions showed a good ability to modeling complex wavelet coefficients [3] [4]. The combination of these models with the Kullbak-Leibler divergence outperforms the GGD based approach in the context of texture retrieval. In this paper, our aim is to propose a more generic model than the previous ones devoted to the complex coefficient magnitudes distribution modeling. We propose a three-parameter Generalized Gamma (GG) distribution, as a

\footnotetext{
${ }^{0}$ This work was supported by franco-morrocan CNRS-CNRST project STIC $05 / 10$
}

flexible model including many distributions, especially, the Weibull and Gamma distributions. The interpretation and use of the GG modeling are discussed, and we present a texture classifier using both the GGD model and the GG one. To measure the similarity between training and testing textures, in the classification framework, we propose the KullbackLeibler divergence, which measures the discrepancy according to the model. The outline of this paper is as follows, in the next section, we give a definition to the Generalized Gamma density and associated Kullback-Leibler divergence. In the third section we present the texture classification framework, while experimental results, are presented in section 4 , which is followed by conclusions.

\section{Genralized Gamma Density For Texture MODELING}

As we have already mentioned, the Generalized Gamma distribution is a generic model, which is suitable for duration analysis, and thus, very useful in different fields [5]. The probability density function of the Generalized Gamma distribution is:

$f(y ; \alpha, \tau, \lambda)=\frac{\tau}{\lambda^{\alpha \tau} \Gamma(\alpha)} y^{\alpha \tau-1} \exp ^{-\left(\frac{y}{\lambda}\right)^{\tau}}, y \geq 0, \alpha, \tau, \lambda>0$

Where $\alpha$ and $\tau$ are shape parameters, $\lambda$ is the scale parameter, and $\Gamma$ (.) is the gamma function. The Generalized Gamma family encompasses a variety of well-known models as limiting distributions. It provides the Gamma density for $\tau=1$, Weibull for $\alpha=1$, and Exponential for $\alpha=\tau=1$. We use the maximum likelihood method for estimating the GG parameters, supposing $y=\left(y_{1}, y_{2}, \ldots, y_{M}\right)$, a set of $\mathrm{M}$ independent coefficients. Let us define the maximum likelihood function of the sample $y: L(y ; \alpha, \tau, \lambda)=\log \prod_{i=1}^{M} f(y ; \alpha, \tau, \lambda)$

$\frac{\partial L(y ; \alpha, \tau, \lambda)}{\partial \alpha}=-M(\tau \log \lambda-\psi(\alpha))+\sum_{i=1}^{M} \tau \log y_{i}=0$

Where $\psi(z)=\Gamma^{\prime}(z) / \Gamma(z)$, is the digamma function. 


$$
\begin{gathered}
\frac{\partial L(y ; \alpha, \tau, \lambda)}{\partial \tau}=M\left(\frac{1}{\tau}-\alpha \log \lambda\right)+ \\
\sum_{i=1}^{M} \alpha \log y_{i}-\left(\frac{y_{i}}{\lambda}\right)^{\tau} \log \frac{y_{i}}{\lambda}=0 . \\
\frac{\partial L(y ; \alpha, \tau, \lambda)}{\partial \lambda}=-\frac{M \alpha \tau}{\lambda}+\frac{\tau \lambda^{-\tau}}{\lambda} \sum_{i=1}^{M} y_{i}=0 .
\end{gathered}
$$

Thus, the parameters are deduced by solving a system of three equations:

$$
\begin{gathered}
\hat{\lambda}=\left[\frac{1}{M \hat{\alpha}} \sum_{i=1}^{M} y_{i} \hat{\tau}\right]^{\frac{1}{\tau}} . \\
\hat{\alpha}=\frac{1}{\hat{\tau}}\left[\frac{\sum_{i=1}^{M} y_{i} \hat{\tau} \log y_{i}}{\sum_{i=1}^{M} y_{i} \hat{\tau}}-\log y_{i}\right]^{-1} . \\
\log \frac{M \hat{\alpha}\left(\prod_{i=1}^{M} y_{i}\right)^{\frac{\hat{\tau}}{M}}}{\sum_{i=1}^{M} y_{i} \hat{\tau}}-\psi(\hat{\alpha})=0 .
\end{gathered}
$$

To make up for the high non-linearity of the system, we use the numerical algorithm of Cohen et al.[6].

Figure 1, shows the fitting of our GG pdf to DTCWT subband coefficient histogram, compared to Weibull and Gamma fitting.

The Similarity Measurement is a crucial step in the classification process. Thus, we need an efficient distance between the images of our database. We adopt the Kullback Leibler divergence which is fully defined by the model parameters of the images. The Kullback Leibler divergence between two PDFs $p\left(X ; \theta_{q}\right)$ and $p\left(X ; \theta_{i}\right)$ is:

$$
K L D\left(p\left(X ; \theta_{q}\right) \| p\left(X ; \theta_{i}\right)\right)=\int p\left(X ; \theta_{q}\right) \log \frac{p\left(X ; \theta_{q}\right)}{p\left(X ; \theta_{i}\right)} d x
$$

By inserting Eq. (1) into Eq. (8), we derive the KLD between two GG PDFs:

$K L D\left(f_{1}\left(. ; \alpha_{1}, \tau_{1}, \lambda_{1}\right), f_{2}\left(. ; \alpha_{2}, \tau_{2}, \lambda_{2}\right)\right)=\log \left(\frac{\tau_{1} \lambda_{2}^{\alpha_{2} \tau_{2}} \Gamma\left(\alpha_{2}\right)}{\tau_{2} \lambda_{1}^{\alpha_{2} \tau_{2}} \Gamma\left(\alpha_{1}\right)}\right)+$

$$
+\left(\frac{\lambda_{1}}{\lambda_{2}}\right) \frac{\Gamma\left(\alpha_{1}+\frac{\tau_{2}}{\tau_{1}}\right)}{\Gamma\left(\alpha_{1}\right)}+\frac{\psi\left(\alpha_{1}\right)}{\tau_{1}}\left(\alpha_{1} \tau_{1}-\alpha_{2} \tau_{2}\right)-\alpha_{1}
$$

So, two GG PDFs are similar to each other when the KLD between them is small.

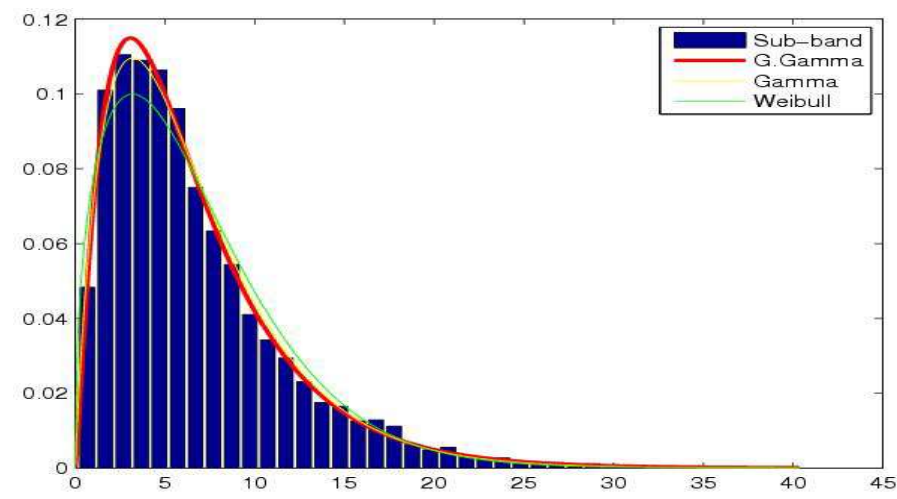

Figure 1. Wavelet subband coefficient magnitude histogram fitted with Generalized Gamma, Weibull and Gamma densities. Example for the Bark.0000.05 subimage. The estimated parameters are: $\alpha=3.1, \tau=1.46$ and $\lambda=0.77$ for GG, $\alpha=7.45$ and $\beta=1.44$ for Weibull, $\alpha=1.95$ and $\beta=3.44$ for Gamma

\section{WAVELET DeCOMPOSITION}

To compensate the lacks of the Discrete Wavelet Transform (DWT), we vote for a complex transformation, specifically the Dual Tree Complex Wavelet Transform (DTCWT). The mean idea here is to calculate the complex transform of signals using two filter's trees A and B (two separate DWT decompositions), that independently generate the real and imaginary coefficients (Figure 2). Contrary to the classic DWT, DTCWT don't suffer of the problems of shift invariance and directional selectivity, providing six orientations at each decomposition level.

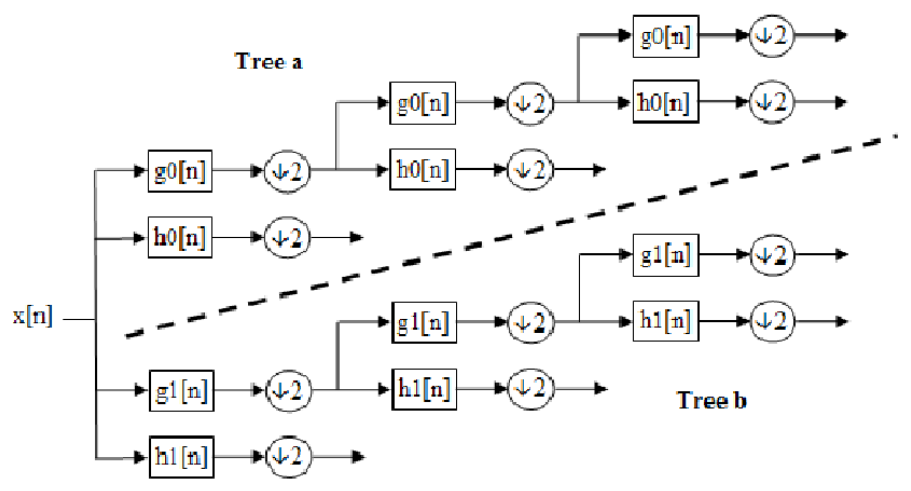

Figure 2. Dual trees for complex wavelet transform

\section{Texture Classification}

Efficient texture classification reposes on pertinent feature extraction and on the choice of well-suited classifier. More precisely, texture classification process is based on a learning and a recognition phases. Firstly, we extract the features for a known set of textures, and we choose a classifier to learn this set, this is called the learning phase. In the recognition phase, an unknown texture is classified by the chosen classifier. 


\section{A. Feature extraction}

Each texture of the database is decomposed via the Dual Tree Complex Wavelet Transform introduced by N.Kingsbury [2]. We choose two scale decomposition $\mathrm{Nsc}=2$, and six orientations Nor $=6$ in each scale. So we may have twelve subbands for each texture. The histograms of the coefficients magnitudes are then modeled by the Generalized Gamma distribution, and the signature (feature vector) of a texture is composed by concatenation of the estimated parameters: $v_{G G}=\left\{\alpha_{1}, \tau_{1}, \lambda_{1}, \ldots, \alpha_{12}, \tau_{12}, \lambda_{12}\right\}$

\section{B. Classifier}

We adopt the K-nearest-neighbor (KNN) classifier, as a simple and good reference in texture classification [7]. KNN is a type of Instance-based classifiers. The mean idea of this approach is that an unknown instance can be done by relating the unknown according to some similarity measure. In our case the Kullback-Leibler divergence is chosen as a similarity measure. The training samples are the feature vectors obtained after the feature extraction step, associated to a class label vectors. In the classification (recognition) phase, an unknown sample is classified by assigning the label of the class to which the majority of its $\mathrm{K}$ nearest neighbor belongs; it's a sort of majority voting.

\section{EXPERIMENTAL RESULTS}

This section aims to show the effectiveness of the Generalized Gamma and DTCWT based approach in the framework of texture classification. We conduct our experiments using the setup presented in [8], considering 30 textures of size $512 \times 512$ from the Vistex database [9], as it's shown in the Figure 3. Each image, is splited into sixteen Sub-images of size $128 \times 128$, so then we obtain a database of 480 samples. Before anything else, we construct a training and testing sets for the classification process. We will, randomly, choose Q samples from each of the 30 texture classes, this will be considered as the training set. Testing set is the rest of the samples. The classification accuracy is obtained by varying Q from 2 to 8 , repeating the KNN test 100 times and then returning the average performance. Firstly, let's point out the flexibility of the Generalized Gamma model in relation to his limiting cases, in the context of texture classification. Table 1 shows the average classification rate vs. the number of training samples for Generalized Gamma, Weibull and Gamma models. We can see the superiority of Generalized Gamma model's classification rates, which is due to its high flexibility.

Secondly, to demonstrate the efficiency of our approach, we compare it to the one based on Generalized Gaussian Density (GGD). This approach was proposed by Woower et al. [8], they used the same KNN classifier using an L2-norm distance as similarity measure.

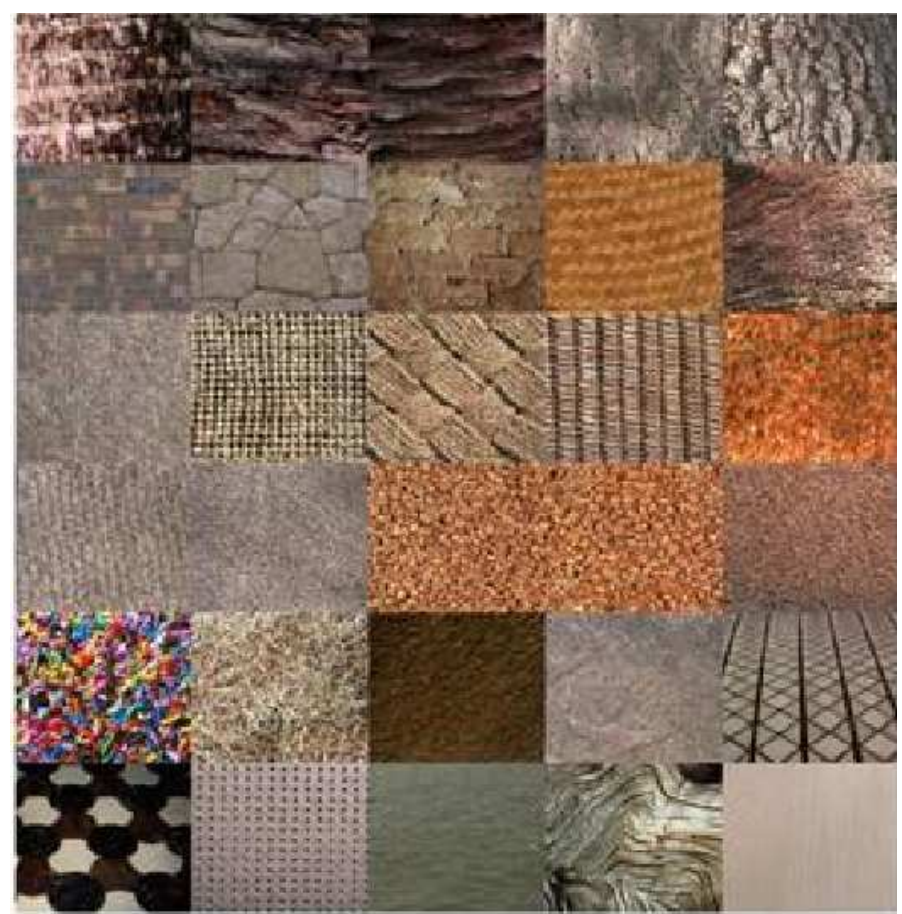

Figure 3. Thirty texture images: From left-right and top bottom: Bark.0009, Bark.0004, Bark.0006, Bark.0008, Bark.0009, Brick.0001, Brick.0004, Brick.0005, Fabric.0000, Fabric.0004, Fabric.0007, Fabric.0009, Fabric.0011, Fabric.0013, Fabric.0016, Fabric.0017, Fabric.001, Food.0000, Food.0002, Food.0005, Food.0008, Grass.0001, Sand.0000, Stone.0004, Tile.0001, Tile.0003, Tile.0007, Water.0006, Wood.0001, Wood.0002

Table I

AVERAGE CLASSIFICATION RATE VS. NUMBER OF TRAINING SAMPLES FOR GENERALIZED GAMMA, WEIBULL AND GAMMA MODELS

\begin{tabular}{|c|c|c|c|c|c|c|c|}
\hline Q & 2 & 3 & 4 & 5 & 6 & 7 & 8 \\
\hline GG & 85.63 & 88.65 & 91.15 & 92.43 & 93.34 & 94.85 & 96.33 \\
\hline Weibull & 84.88 & 87.29 & 89.56 & 91.25 & 92.44 & 93.16 & 95.08 \\
\hline Gamma & 84.19 & 86.73 & 88.96 & 91.33 & 91.84 & 92.76 & 94.42 \\
\hline
\end{tabular}

Table II

AVERAGE CLASSIFICATION RATE VS. NUMBER OF TRAINING SAMPLES FOR GENERALIZED GAMMA AND GGD

\begin{tabular}{|c|c|c|c|c|c|c|c|}
\hline Q & 2 & 3 & 4 & 5 & 6 & 7 & 8 \\
\hline GG & 85.63 & 88.65 & 91.15 & 92.43 & 93.34 & 94.85 & 96.33 \\
\hline GGD & 83.9 & 85.37 & 88.31 & 89.26 & 91.12 & 91.96 & 92.41 \\
\hline
\end{tabular}

The results, of the 100 random experiments (Table 2), show that our approach achieves the highest average classification rate $96.33 \%$ for $\mathrm{Q}=8$ ( $\mathrm{nb}$. of training samples from each class), while the GGD-based approach obtain $92.41 \%$. Finally, we will focus on the suitability of the DT-CWT decomposition with the Generalized Gamma based approach. For this purpose, we shall compare it to the steerable pyramid decomposition [GG+steerpyr] performances [10]. Table 3 points out the choice of the decomposition; we compare the same model (GG) in combination with 
the two decompositions. We observe a higher performance of the DTCWT based approach, in term of higher average classification rate.

Table III

AVERAGE CLASSIFICATION RATE VS. NUMBER OF TRAINING SAMPLES FOR GENERALIZED GAMMA IN COMBINATION WITH DTCWT AND STEERABLE PYRAMIDS

\begin{tabular}{|c|c|c|c|c|c|c|c|}
\hline Q & 2 & 3 & 4 & 5 & 6 & 7 & 8 \\
\hline GG+DTCWT & 85.63 & 88.65 & 91.15 & 92.43 & 93.34 & 94.85 & 96.33 \\
\hline GG+Steerpyr & 80.02 & 83.82 & 85.89 & 88.28 & 90.89 & 91.01 & 93.22 \\
\hline
\end{tabular}

\section{CONCLUSION}

This paper presents, a statistical approach for texture classification, using the Generalized Gamma Density (GG), and the Dual Tree Complex Wavelet Transform decomposition. The Generalized Gamma Density has the advantage to be a more flexible and a generic model. Each, texture of the database, was decomposed via the DTCWT decomposition, and the histograms of the magnitudes of the subbands coefficients are then modeled using the GG density, which the parameters serve as a features for the texture classification process. A KNN classifier is designed for classifying the unknown textures. The experiments, on a thirty class of the VisTex database, show that the combination of the Generalized Gamma Density and the DTCWT gives good performances over other models and decompositions. In future work, we study the performance of the Generalized Gamma Density in combination with the Dual Tree M-band Wavelet Transform.

\section{REFERENCES}

[1] M. N. Do and M. Vetterli, "Wavelet-Based Texture Retrieval Using Generalized Gaussian Density and Kullback-Leibler Distance” IEEE Trans. Image Process. vol. 11, no. 2, pp. 146158, Feb 2002.

[2] N. Kingsbury, "The Dual-Tree Complex Wavelet Transform: A new Technique for Shift-Invariance and Directional Filters," in Proceedings of the 8th IEEE DSP Workshop, Bryce Canyon, Utah, USA, Aug. 1998, pp. 9-12.

[3] R.Kwitt and A.Uhl, "Image similarity measurement by Kullback-Leibler divergences between complex wavelet subband statistics for texture retrieval", in Proceedings of the IEEE international Conference on Image Processing (ICIP' 2008), Oct. 2008, pp.933-936.

[4] J. Mathiassen, A. Skavhaug, and K. B, "Texture Similarity Measure Using Kullback-Leibler Divergence between Gamma Distributions," European Conference on Computer Vision 2002, 2002, pp. 19-49.

[5] E. W. Stacy, "A Generalization of the Gamma Distribution" Ann. Math. Statist., vol. 33, 1962., pp. 1187-1192.

[6] Cohen and Whittle, (1988) "Parameter Estimation in Reliability and Life Span Models", p. 220 ff, Marcel Dekker.
[7] K. Fukunaga, "Introduction to Statistical Pattern Recognition" Academic Press, New York, 1972.

[8] Van de Wouver, G.Scheunders, P.Van Dyck: "Statistical texture characterization from discrete wavelet representation". IEEE Trans. Image Process. 8(4), 592-598 (1999).

[9] "MIT vision and modeling group," [Online], Available from: http://vismod.www.media.mit.edu.

[10] E. P. Simoncelli and W. T. Freeman, "The Steerable Pyramid: A Flexible Architecture for Multi-Scale Derivative Computation," Proc. 2nd Int'l Conf. Image Process., Washington DC, vol. III, pp. 444-447, Oct 1995. 